\title{
Fabrication and Characterization of Dye-sensitized Solar Cells based on Anodic Titanium Oxide Nanotube Arrays Sensitized with Heteroleptic Ruthenium Dyes
}

\author{
Chien-Hung Shen, Yu-Cheng Chang, Po-Ting Wu and Eric Wei-Guang Diau*
}

Department of Applied Chemistry and Institute of Molecular Science, National Chiao Tung University, Hsinchu 30010, Taiwan

\begin{abstract}
Anodic self-organized titania nanotube (TNT) arrays have a great potential as efficient electron-transport materials for dye-sensitized solar cells (DSSC). Herewith we report the photovoltaic and kinetic investigations for a series of heteroleptic ruthenium complexes (RD16-RD18) sensitized on TNT films for DSSC applications. We found that the RD16 device had an enhanced short-circuit current density $\left(J_{\mathrm{SC}} / \mathrm{mA} \mathrm{cm}=15.0\right)$ and an efficiency of power conversion $(\eta=7.2 \%)$ greater than that of a N719 device $(\eta=7.1 \%)$ due to the increasing light-harvesting and the broadened spectral features with thiophene-based ligands. However, the device made of RD17 (adding one more hexyl chain) showed smaller $J_{\mathrm{SC}}\left(14.1 \mathrm{~mA} \mathrm{~cm} \mathrm{~cm}^{-2}\right)$ and poorer $\eta(6.8 \%)$ compare to those of RD16 due to smaller amount of dye-loading and less efficient electron injection for the RD17 device than for the RD16 device. For the RD18 dye (adding one more thiophene unit and one more hexyl chain), we found that the device showed even lower $J_{\mathrm{SC}}\left(13.2 \mathrm{~mA} \mathrm{~cm}{ }^{-2}\right)$ that led to a poorest device performance $(\eta=6.2 \%)$ for the RD18 device. These results are against to those obtained from the same dyes sensitized on $\mathrm{TiO}_{2}$ nanoparticle films and they can be rationalized according to the electron transport kinetics measured using the methods of charge extraction and transient photovoltage decays.
\end{abstract}

Dye-sensitized solar cells (DSSC) have received much attention because of the demand for cheap sources of renewable energy. ${ }^{1-3}$ The efficiency of power conversion $(\eta)$ of Ru-based dyes using a traditional nanoparticle (NP)-based $\mathrm{TiO}_{2}$ photoanode

*To whom correspondence should be addressed.

E-mail: diau@mail.nctu.edu.tw attained $11 \%{ }^{4-6}$ while that of a porphyrin-based DSSC has exceeded $12.3 \%$ under one-sun illumination. ${ }^{7}$ To enhance the light-harvesting ability of the dye, well-ordered one-dimensional $\mathrm{TiO}_{2}$ nanotube (TNT) arrays become effective candidates for photoanode materials because of their native light-scattering effect, superior electron transport and small rate of charge recombination relative to a conventional NP-based system. $^{8-10}$ However, a traditional NT-DSSC device based on illumination of the back side suffers degradation of performance because some incident light is absorbed by the iodide/tri-iodide electrolyte and scattered by the Pt-coated counter electrode. Therefore, we designed heteroleptic ruthenium complexes containing benzimidazole (BI) ligands with thiophene derivatives attached on the pyridine part of the ancillary ligands to improve the light-harvesting efficiency via enhancing the absorption coefficient of the dye. ${ }^{11-13}$ In particular, the NP-based devices made of RD16-RD18 dyes (the corresponding molecular structures are shown in Figure 1) exhibited systematic enhanced short-circuit current density $\left(J_{\mathrm{SC}}\right)$ and $\eta$ due to the increasing light-harvesting ability and the broadened spectral features with thiophene-based ligands. $^{13}$ In the present study, we carried out photovoltaic and transient photoelectric characterizations for this series of RD dyes sensitized on TNT films (the corresponding SEM images are shown in Figure 2). We found that the device performance of these RD-based NT-DSSC exhibited a trend that is opposite to their NP-based counterpart. Incident photo-to-current conversion efficiency (IPCE), amount of dye-loading (DL), charge extraction (CE) and transient photovoltage decay (TVD) measurements were performed to rationalize the observed trend of device performance. ${ }^{14}$ 
The dyes of the RD series (RD16-RD18) were synthesized according to a procedure reported elsewhere; ${ }^{13}$ dye N719 was purchased (Everlight Chemical, Taiwan). The $\mathrm{TiO}_{2}$ NT films with tube length $\sim 25 \mu \mathrm{m}$ were produced via a typical anodization procedure reported elsewhere. ${ }^{8-10}$ The NT films were treated with $\mathrm{TiCl}_{4}(0.09 \mathrm{M})$ and immersed in dye solutions $(0.3 \mathrm{mM}$ in anhydrous $\mathrm{CH} 3 \mathrm{CN} / t-\mathrm{BuOH} \quad(1: 1 \mathrm{v} / \mathrm{v})$ at $25{ }^{\circ} \mathrm{C}$ for $\left.1 \mathrm{~h}\right)$ containing CDCA $(0.9 \mathrm{mM})$ for dye loading onto the working electrodes. The counter electrode was made on spin-coating the $\mathrm{H}_{2} \mathrm{PtCl}_{6}$ /isopropyl alcohol solution onto a FTO glass substrate. The electrolyte solution contained butylmethyl-imidazolium iodide (BMII, 0.6 $\mathrm{M})$, guanidinium thiocyanate (GuSCN, $0.1 \mathrm{M})$, LiI $(0.1 \mathrm{M}), \mathrm{I}_{2}(0.01 \mathrm{M})$, and 4-t-butylpyridine (TBP, 0.5 $\mathrm{M})$ in acetonitrile. The current-voltage characteristics were determined with a digital source meter (Keithley 2400) with the device under one-sun AM $1.5 \mathrm{G}$ irradiation from a solar simulator (XES-502S, SAN-EI). The spectra of the IPCE of the corresponding devices were recorded with a system comprising a Xe lamp (PTi A-1010, 150 W), a monochromator (PTi, $1200 \mathrm{gr} \mathrm{mm}^{-1}$ blazed at 500 $\mathrm{nm}$ ), and a source meter (Keithley 2400). Charge densities $(\mathrm{Ne})$ were determined via the $\mathrm{CE}$ method and electron lifetimes $\left(\tau_{R}\right)$ via the TVD method for the NT-DSSC devices made of RD16-RD18 dyes under seven light intensities; the details on device characterization using the CE and TVD methods are given elsewhere. ${ }^{14}$

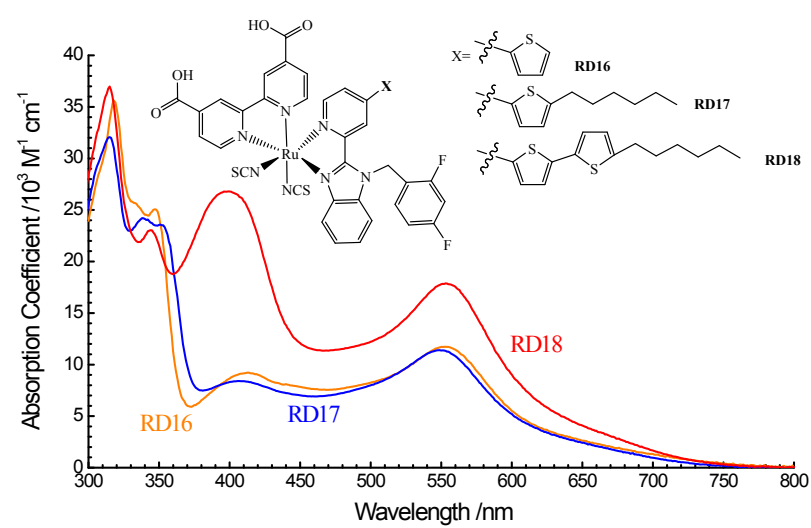

Figure 1. Absorption coefficient spectra of RD16, RD17 and RD18 in DMF (adopted from ref. 13) and molecular structures of RD16, RD17 and RD18 with $\mathrm{X}$ representing the corresponding thiophene substituents.
Figure 1 shows the absorption spectra of the RD16, RD17 and RD18 dyes in DMF solution. Indicated in these spectra, thiophene substituents effectively improved the absorption coefficients for these dyes with a systematic order RD18 $>$ RD17 $\sim$ RD16, whereas the hexyl substitute plays no role in enhancing the absorption coefficients. In a previous study for RD16-RD18 sensitized on $\mathrm{TiO}_{2} \mathrm{NP}$ films, ${ }^{13}$ we found that the device performance exhibited a systematic trend with the order RD18> RD17> RD16, consistent with the trend of the absorption spectra of this series of dyes. In the present investigation, these dyes were sensitized inside the TNT arrays to form the NT-DSSC devices. Figure 2 presents top and cross-section views of SEM images of the $\mathrm{TiO}_{2}$ NT arrays and shows the nano-channels and pores with a diameter of $140 \mathrm{~nm}$ and a 25 nm-thick pore wall; the tube length (film thickness) is $\sim 25 \mu \mathrm{m}$. The photovoltaic results of these RD dyes sensitized inside these TNT arrays are discussed in the following.

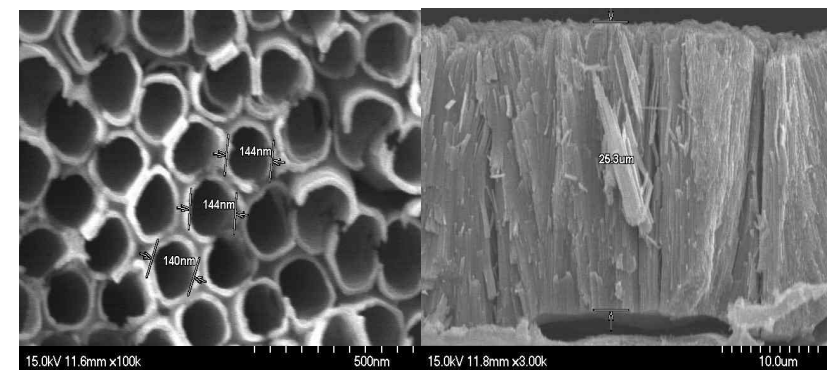

Figure 2. Top-view (left) and side-view (right) SEM images of the $\mathrm{TiO}_{2} \mathrm{NT}$ filmutilized as photoanode for RD-based NT-DSSC devices.

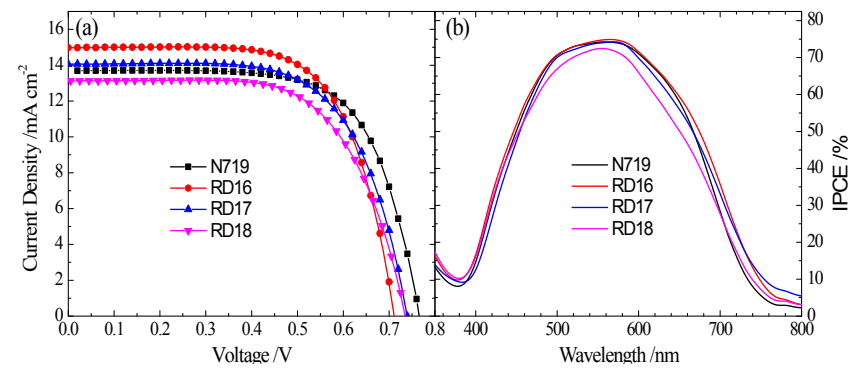

Figure 3. Photovoltaic properties: (a) current-voltage characteristics and (b) the corresponding IPCE action spectra of devices made of the N719, RD16, RD17and RD18 dyes with the same fabricated TNT films (thickness $25 \mu \mathrm{m}$ ). 
Table 1. Photovoltaic parameters of DSSC with photosensitizers $\mathrm{N} 719$ and RD16-RD18, and under AM-1.5G one-sun illumination (power $100 \mathrm{~mW} \mathrm{~cm}^{-2}$ ) and active area $0.16 \mathrm{~cm}^{2}$

\begin{tabular}{cccccc}
\hline Dye & $\begin{array}{c}J_{\mathrm{SC}} / \\
\mathrm{mA} \mathrm{cm}\end{array}$ & $\begin{array}{c}V_{\mathrm{OC}} / \\
\mathrm{V}\end{array}$ & FF & $\begin{array}{c}\eta / \\
\%\end{array}$ & $\begin{array}{c}\mathrm{DL} / \\
\mathrm{nmol} \mathrm{cm}\end{array}$ \\
\hline $\mathrm{N} 719$ & 13.69 & 0.768 & 0.680 & 7.1 & 260 \\
$\mathrm{RD} 16$ & 14.98 & 0.714 & 0.671 & 7.2 & 210 \\
$\mathrm{RD} 17$ & 14.06 & 0.744 & 0.649 & 6.8 & 190 \\
$\mathrm{RD} 18$ & 13.15 & 0.738 & 0.644 & 6.2 & 150 \\
\hline
\end{tabular}

Figures $3 \mathrm{a}$ and $3 \mathrm{~b}$ show typical current-voltage characteristics and the corresponding IPCE action spectra, respectively, for the devices made of each ruthenium dye. Table 1 summarizes the amounts of DL and the photovoltaic parameters. The photovoltaic performances of these devices show a systematic trend of $J_{\mathrm{SC}}$ with the order $\mathrm{RD} 16>\mathrm{RD} 17>\mathrm{N} 719$ (reference) $>\mathrm{RD} 18$, which is against to the trend of $J_{\mathrm{SC}}$ observed in the corresponding NP-based DSSC. ${ }^{13}$ The trend of DL seems to agree with the trend of $J_{\mathrm{SC}}$ for the RD dyes but this cannot explain the fact that RD18 has much greater absorption coefficients shown in Figure 1. The IPCE spectra shown in Figure $3 b$ indicate that the RD18 device had lower IPCE values over the entire spectrum compared to those of RD16 and RD17 devices. The effect of $J_{\mathrm{SC}}$ on alkyl substitution between RD16 and RD17 was also negative, giving the $J_{\mathrm{SC}}$ value of the RD16 device reaching $15.0 \mathrm{~mA} \mathrm{~cm}^{-2}$. On the other hand, the $V_{\mathrm{OC}}$ values of the devices show an order N719 $>$ RD17 $>$ RD18 $>$ RD16. This trend indicates that an involvement of the thiophene group in the BI ligand decreased the value of $V_{\mathrm{OC}}$, but an additional long alkyl chain at the end of the thiophene group helped to promote $V_{\mathrm{OC}}$ slightly. As a result, the overall device performances show a trend RD16 $(\eta=7.2$ $\%)>\operatorname{N719}(\eta=7.1 \%)>\operatorname{RD} 17(\eta=6.8 \%)>$ RD18 $(\eta=6.2 \%)$; this trend in NT-DSSC is against to the trend in NP-DSSC, which would be explained by the results obtained from the CE/CVD measurements discussed below.

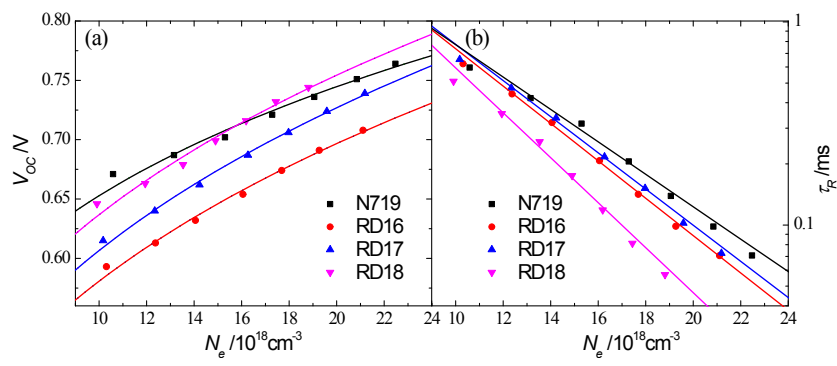

Figure 4. (a) Open-circuit voltage $\left(V_{\mathrm{OC}}\right)$ and (b) electron lifetimes $\left(\tau_{R}\right)$ as a function of extracted charge densities $(\mathrm{Ne})$ for devices made of N719 and RD16-RD18 irradiated with bias light at eight intensities.

Figures $4 \mathrm{a}$ and $4 \mathrm{~b}$ display plots of $V_{\mathrm{OC}}$ vs $N \mathrm{e}$ and $\tau_{R}$ vs $N$ e, respectively, for the four devices irradiated with white light from a LED at seven intensities. The $\mathrm{CE}$ results (Figure 4a) indicate that the positions of the $\mathrm{TiO}_{2}$ potentials of the devices have the order N719 $>$ RD18 $>$ RD17 > RD16, whereas the CVD results (Figure 4b) show the electron lifetimes of the devices having the order N719 $>$ RD17 $\sim$ RD16 $>$ RD18. We can easily understand that $V_{\mathrm{OC}}$ of the N719 device is greater than those of the other RD devices because (i) the CB edge of the TNT film sensitized with N719 was up-shifted more than the other devices and (ii) the rate of charge recombination in the N719 device was smaller than those of the other $\mathrm{RD}$ devices. The lowest $V_{\mathrm{OC}}$ value for the RD16 device can be understood being due to the $\mathrm{CB}$ edge of the TNT potential, which was significantly down-shifted for the RD16 device than for those of the other devices as shown in Figure 4a. For the RD18 device, even though the TNT potential was higher than those of the other RD devices, the rapid charge recombination in the interface between the RD18/TNT film and the electrolyte made the $V_{\mathrm{OC}}$ value of RD18 becoming smaller than that of the RD17 device.

For the functionality of these $\mathrm{RD}$ dyes, the BI-based ancillary ligands seem to have an effect of lowering the potential to some extent, and also accelerating the charge recombination for the observed lower $V_{\mathrm{OC}}$ for $\mathrm{RD}$ devices than for the N719 device. Added a further hexyl chain at the thiophene end of the ligand decreased slightly the potential but helps to retard the charge recombination to account for the $V_{\mathrm{OC}}$ of RD17 being larger than that of RD16; adding a second thiophene unit into the ligand of $\mathrm{RD} 17$ to form RD18 slightly raised the potential but accelerated charge recombination, giving the $V_{\mathrm{OC}}$ value being smaller for the RD18 device than for the RD17 device. Our results thus indicate that the alkyl chain and the thiophene units inside the TNT arrays affected the potential more for the RD16 dye and affected the charge recombination more for the RD18 dye. Because of the effect of accelerated charge recombination for the thiophene substituent, we expect that the charge collection efficiency in the RD18 device was smaller than those in the RD16 and RD17 devices, consistent with the trend shown in the IPCE spectra (Figure 3b). The greater $J_{\mathrm{SC}}$ of the RD16 device than those of the other devices might be due to the lower $\mathrm{CB}$ potential 
of the TNT film giving a superior electron injection for RD16 than for other RD dyes.

In conclusion, we studied a series of heteroleptic ruthenium complexes with the benzimidazole (BI) ligands containing varied thiophene units (RD16-RD18) sensitized on films of oriented anodic titania nanotube arrays for dye-sensitized solar cells. In our previous investigations based on $\mathrm{TiO}_{2}$ nanoparticle films, ${ }^{13}$ we found that increasing the number of thiophene units in the BI ancillary ligands enhanced the light-harvesting ability to give $J_{\mathrm{SC}}$ and the corresponding IPCE of the devices with the order RD18 $>$ RD17 > RD16> N719, and $V_{\mathrm{OC}}$ of the devices showing the order N719 $>$ RD17 > RD18 $\sim$ RD16. However, in the present study the trend of device performances on TNT-based DSSC is opposite: $J_{\mathrm{SC}}$ shows the order $\mathrm{RD} 16>\mathrm{RD} 17>\mathrm{N} 719>\mathrm{RD} 18$ and $V_{\mathrm{OC}}$ shows the order N719 $>$ RD17 $>$ RD18 $>$ RD16. The variation of $V_{\mathrm{OC}}$ is understood from the results of $\mathrm{CE}$ and TVD measurements: the addition of one thiophene unit in RD16 down shifted the potential, but adding alkyl chain in RD17 and one more thiophene unit in RD18 up-shifted the potential; charge recombination seems to be retarded by adding the alkyl chain in RD17 but it was accelerated by adding one more thiophene unit in RD18. The great $J_{\mathrm{SC}}$ of the RD16 device might be due to the effect of potential down shift, which gives the overall power conversion efficiency $\eta=7.2 \%$ for the NT-DSSC device in a back-illumination structure.

Received January 14, 2014; Accepted March 4, 2014

KEYWORDS: Dye-sensitized solar cells, Ruthenium complexes, Heteroleptic ligands, $\mathrm{TiO}_{2}$ nanotube arrays

\section{ACKNOWLEDGEMENT}

National Science Council of Taiwan and Ministry of Education of Taiwan, under the ATU program, provided financial support for this project.

\section{REFERENCES AND NOTES}

1. Grätzel, M. Nature, 2001, 414, 338-344.

2. Gratzel, M. Acc. Chem. Res. 2009, 42, $1788-1798$.

3. Hagfeldt, A.; Boschloo, G.; Sun, L.; Kloo, L.; Pettersson, H. Chem. Rev. 2010, 110, 6595-6663.

4. Nazeeruddin, M. K.; De Angelis, F.; Fantacci, S.; Selloni, A.; Viscardi, G.; Liska, P.; Ito, S.; Takeru, B.; Gratzel, M. J. Am. Chem. Soc. 2005, 127, $16835-16847$.

5. Wang, Q.; Ito, S.; Gratzel, M.; Fabregat-Santiago, F.; Mora-Sero, I.; Bisquert, J.; Bessho, T.; Imai, H. J. Phys. Chem. B 2006, 110, 25210-25221.

6. Mor, G. K.; Shankar K. ; Paulose, M.; Varghese, O. K.; Grimes, C. A. Nano Lett., 2006, 6, 215.

7. Yella, A.; Lee, H. W.; Tsao, H. N.; Yi, C.; Chandiran, A. K.; Nazeeruddin, M. K.; Diau, E. W.-G.; Yeh, C. Y.; Zakeeruddin, S. M.; Grätzel, M. Science 2011, 334, 629-634.

8. Chen, C.-C.; Chung, H.-W.; Chen, C.-H.; Lu, H.-P.; Lan, C.-M.; Chen, S.-F.; Luo, L.-Y.; Hung C.-S.; Diau, E. W.-G, J. phys. Chem. C, 2008, 112, 19151-19157.

9. Li, L.-L.; Tsai, C.-Y.; Wu, H.-P.; Chen, C.-C.; Diau, E. W.-G. J, Mater. Chem., 2010, 20, 2753-2758.

10. Li, L.-L.; Chen, Y.-J.; Wu, H.-P.; Wang, N. S. and Diau, E. W.-G. Energy Environ. Sci., 2011, 4, 3420-3425.

11. Huang, W.-K.; Cheng, C.-W.; Chang, S.-M.; Lee, Y.-P.; Diau, E. W.-G. Chem. Commun. 2010, 46, 8992-8995

12. Huang, W.-K.; Wu, H.-P.; Lin, P. L.; Lee, Y.-P.; Diau, E. W.-G. J. Phys. Chem. Lett. 2012, 3, 1830-1835.

13. Huang, W.-K.; Wu, H.-P.; Lin, P.-L.; Diau, E. W.-G. J. Phys. Chem. C 2013, 117, 2059-2065.

14. Li, L.-L.; Chang, Y.-C.; Wu, H.-P.; Diau, E. W.-G. Int. Rev. Phys. Chem., 2012, 31, 420-467. 